\title{
Foot Strike Patterns Differ Between Children and Adolescents Growing up Barefoot vs. Shod
}

(ब) (1) $\odot$

Authors

Karsten Hollander ${ }^{1}$, Johanna Elsabe de Villiers ${ }^{2}$, Ranel Venter ${ }^{2}$, Susanne Sehner ${ }^{3}$, Karl Wegscheider ${ }^{3}$, Klaus-Michael Braumann', Astrid Zech ${ }^{4}$

\author{
Affiliations \\ 1 University of Hamburg, Institute of Human Movement \\ Science, Department of Sports and Exercise Medicine, \\ Hamburg, Germany \\ 2 Stellenbosch University, Department of Sport Science, \\ Matieland, South Africa \\ 3 University Medical Center Hamburg-Eppendorf, \\ Department of Medical Biometry and Epidemiology, \\ Hamburg, Germany \\ 4 University of Jena, Department of Human Movement \\ Science and Exercise Physiology, Jena, Germany
}

\section{Key words}

habitual barefoot, running, biomechanics, footwear, rear foot strike

accepted 05.09.2017

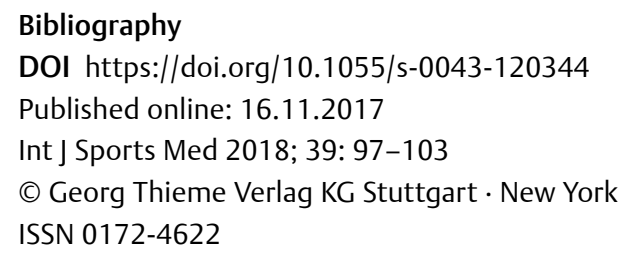

\section{Correspondence}

Dr. Karsten Hollander, MD

University of Hamburg, Institute of Human Movement Science Department of Sports and Exercise Medicine

Turmweg 2

20148, Hamburg

Germany

Tel.: + 49/40/42838 9144, Fax: + 49/40/428386268

karsten.hollander@uni-hamburg.de

\begin{abstract}
Effects of early and permanent footwear use are not well understood. The aim of this study was to investigate the effects of habituation to footwear on foot strike patterns of children and adolescents. Healthy habitually barefoot and shod participants (aged 6-18 years) from South Africa $(n=288)$ and Germany ( $n=390$ ) performed multiple 20 -m jogging and running trials with and without shoes. Each foot strike was captured using a high-speed camera to determine a rearfoot or non-rearfoot strike. The probability of a rearfoot strike in both cohorts and each age was analyzed by using a mixed-effects logistic regression adjusted for possible confounders. Habitually barefoot children showed a higher probability of using rearfoot strikes than habitually shod children $(p<0.001)$. The probability was age-dependent and decreased in habitually barefoot children with age $\left(\mathrm{OR}_{\text {barefoot-jogging }}=0.82,95 \% \mathrm{Cl}, 0.71\right.$ to $0.96, p=0.014 ; \mathrm{OR}_{\text {barefoot-running }}=0.58,95 \% \mathrm{Cl}, 0.50$ to 0.67 , $\mathrm{p}<0.001$ and $\mathrm{OR}_{\text {shod-running }}=0.68,95 \% \mathrm{Cl}, 0.59$ to 0.79 , $\mathrm{p}<0.001)$. In habitually shod children, the probability increased significantly for shod jogging $(\mathrm{OR}=1.19,95 \% \mathrm{Cl}, 1.05$ to 1.35 , $p=0.006)$. To conclude, foot strike patterns of children are influenced by habituation to footwear. Younger habitually barefoot children show higher rates of rearfoot strikes for shod and barefoot running, and it converges in later adolescence.
\end{abstract}

\section{Introduction}

Even though barefoot locomotion has already been used for millions of years [23], it has regained a new scientific and social interest within the last decade. The advantages and disadvantages are widely discussed [32,37], with no generally accepted evidence for long-term effects [16].

It is known that acute transition from shod to barefoot locomotion influences walking and running biomechanics $[6,9]$ and is mostly associated with changes in foot strike patterns (FSP) $[21,25,36]$. When running barefoot, the FSP of adults typically changes from a rearfoot strike (RFS) to a midfoot or forefoot strike, with a more plantarflexed ankle at ground contact [9, 14, 21]. Furthermore, step length is reduced, accompanied by an increased step frequency $[3,6]$. Some studies have found reduced ground reaction forces for barefoot compared to shod running $[9,14,25]$, whereas other studies emphasize the influence of foot strike pat- 
terns on ground reaction forces to be more important than the footwear $[9,35]$. Most of the biomechanical research investigates adult populations, but comparable evidence exists for children and adolescents [17, 29,40]. Although these findings generally support assumptions regarding the strong influence of regular shoe use on the development of individual movement patterns, only limited knowledge exists for the habituation to (simulated) barefoot running [16, 26, 39]. In addition to well-conducted prospective studies, investigating populations habituated to barefoot locomotion and comparing them to habitually shod (HS) populations can be a promising study design to better understand the long-term consequences of barefoot locomotion. Although there are a few such studies investigating FSP of a habitually barefoot (HB) population $[13,24,25,33]$, the effects of habituation to barefoot locomotion on FSP are still inconclusive. Some studies, for example, show higher rates of RFS $[13,33]$ whereas others show a lower rate in HB populations [21, 25]. In addition, Lieberman and colleagues [24] reported a high variation of FSP in a HB population not accustomed to running. Most of the presented studies report solely on adults and a few on adolescents. Childhood is an important phase for motor development, and it remains unclear whether it is valid to extrapolate the knowledge from adults to pediatric populations. Therefore, this study's aim was to compare FSP of HB and HS children and adolescents aged $6-18$ years. Furthermore, the study aims to evaluate the development of FSP during motor development over time.

\section{Methods}

\section{Participants and study design}

For this cross-sectional observational study, children and adolescents from schools across rural and urban areas in the Western Cape, South Africa and Northern Germany were included. Children were recruited in two different regions due to the obligation to wear shoes at school in Germany whereas it is common to attend school barefoot in South Africa. After approval from the responsible school authorities, schools in the regions were randomly selected per stratum and contacted by the research team. When school directors and physical education teachers agreed to participate, information sheets and consent forms were distributed to all children (and parents). Healthy volunteers aged $6-18$ years were eligible for this study if physically active for at least 120 accumulative minutes per week (as reported by their parent(s)).

To determine whether a study participant was eligible as "habitually barefoot”, a barefoot questionnaire was used [18]. This six-item questionnaire was specially developed for this study and uses a three-point Likert scale to determine whether a child was barefoot most of the time/half of the time/none of the time 1) during school, 2 ) during sports and 3 ) in and around the house. Participants were included as HB if they had a score of $\geq 3$ (out of max 6 points), equivalent to being at least half of the time barefoot at school or at sports in addition to being barefoot at home. At least 16 children with an equal distribution of both sexes were recruited in each age. Exclusion criteria consisted of current injuries as well as orthopedic, neurological or neuromuscular abnormalities likely to affect gait. Volunteers with a signed parental consent form were tested at the school during their regular physical education lessons.
Testing took place between March 2015 and June 2016 in 22 primary and secondary schools; the full study protocol has been published elsewhere [18]. Ethical approval was obtained from the ethics committee of the medical association (protocol number PV4971) and the University's ethics committee (protocol number HS1153/2014). The study was conducted in accordance with international ethical standards [11] and reports according to the STROBE guidelines for reporting observational studies [38].

\section{Data measurement and variables}

Prior to the testing period, a joint training of research teams was held in Germany over several days to ensure the identical use of the equipment and data collection. In addition, the investigator leading all testing in Germany $(\mathrm{KH})$ also attended the first weeks of testing in South Africa. The testing protocol for this study consisted of anthropometrical (date of birth, height, weight, sex, ethnicity) and biomechanical measurements, as well as a physical activity questionnaire (PAQ-C and PAQ-A) [19].

\section{Foot strike pattern assessment}

The FSP was determined during 20-m jogging and running trials in barefoot and shod conditions. For jogging, children were asked to go at a comfortable self-selected speed, whereas for the running condition they were asked to run maximally. The order of the starting condition (barefoot or shod) was randomized and the participants used the footwear they normally wear during their physical education class, normally sport shoes. In this field study, we did not measure footwear properties such as cushioning, flexibility and heel-to-toe drop. The running track was leveled and consisted of a hard underground (concrete or hard gym floor covering). To measure velocity, magnetic timing gates (Humotion Smartracks, Münster, Germany) were used in Germany and photoelectric gates (Brower Timing Systems, Salt Lake City, UT, USA) in South Africa [18]. A wide-angle high-speed camera (GoPro HD Hero 4, GoPro Inc., San Mateo, California, USA) was placed $1.5 \mathrm{~m}$ orthogonal to the midline of the running track at the $17.5-\mathrm{m}$ mark of the straight $20-m$ course. The video data was processed (Adobe Premiere Pro CS 6, Adobe Systems, San Jose, California, USA) and rated independently by two reviewers with a third experienced reviewer for consensus. The FSP was rated as RFS (first ground contact with the heel) or non-RFS (first contact with the forefoot or simultaneous ground contact of the forefoot and heel). This grouping was chosen in order to have a dichotomous variable and due to a very high accuracy in determining a rearfoot strike (interrater accordance: 0.981 ) and a lower accuracy in deciding between a forefoot strike and midfoot strike (0.893). This method has already been used successfully in other studies [12, 14, 21]. Despite good evidence for validity of laboratory-based foot strike pattern determination [1,34], to the author's knowledge there is only one study showing video-based assessment of foot strike pattern to be valid [5].

\section{Statistical methods}

Sample characteristics are given as absolute and relative frequencies or mean + /- standard deviation, whichever is appropriate. The probability of RFS was analyzed using a mixed-effects logistic regression, adjusting for the clustered structure induced by repeated measurements by condition (jogging/run and barefoot/shod) and 
a repeat of the run condition per child. The predictors HB (yes/no), age and conditions, as well as their four-way interaction and resulting lower degrees of interactions, were modeled as fixed effects. In the case of an insignificant interaction term, only the lower degrees of interactions or main effects were included. This decision was made by using the likelihood ratio test for model comparison. Moreover, in the model, the following confounders were included: BMI, sex, ethnicity, PAQ score and side. The adjusted results were estimated as marginal means, which were represented as graphs with $95 \%$ confidence intervals $(95 \% \mathrm{Cl})$ as adjusted probability; additionally, the odds ratios with corresponding $95 \%$ confidence intervals were reported. Post hoc tests for comparison of the estimated means were calculated with contrast tests using Wald tests. All of the models present available case analyses. Nominal p-values were reported without correction for multiplicity. A two-tailed $p<0.05$ was considered to be statistically significant. An a priori sample size was conducted and published in the study protocol [18]. All of the analyses were performed using STATA 14 (StataCorp. 2015. Stata Statistical Software: Release 14. College Station, TX: StataCorp LP).

\section{Results}

\section{Participants}

After initial testing of 1017 children and adolescents, 678 children (49.4\% females, $50.6 \%$ males; mean \pm SD age $12.4 \pm 3.22$ years, body height $156.1 \pm 17.3 \mathrm{~cm}$; weight $49.6 \pm 17.6 \mathrm{~kg}$; BMl $19.7 \pm 3.9 \mathrm{~kg} / \mathrm{m}^{2}$; PAQ score $2.9 \pm 0.7$ ) were included and 7445 valid trials were analyzed. Reasons for exclusion were not meeting all inclusion criteria $(n=131)$, missing or incomplete video data $(n=103)$, missing or incomplete questionnaires ( $n=74)$, missing data for confounders $(n=29)$, or refusing to participate $(n=2)$. The rate of HB children in South Africa was $90.9 \%$, whereas all German participants were HS. The demographic data of all included participants and subgroups are presented in > Table $\mathbf{1}$ and Table S1.

\section{Primary analysis: Foot strike pattern and age effect}

The analysis revealed a global interaction effect of the independent variable $\mathrm{HB}$ vs. $\mathrm{HS}$, which depends on age and all investigated conditions (shod/barefoot and jogging/running). All observed (unadjusted) and estimated (adjusted) percentages of RFS for $\mathrm{HB}$ and HS children and adolescents can be found in - Fig. 1. HB children were more likely to adopt a RFS for barefoot conditions. Furthermore, the trend over age differed between both groups in jogging and running conditions independent of footwear $\left(\mathrm{OR}_{\text {run vs jogging }}\right.$ for HB children: $0.70 ; 95 \% \mathrm{Cl}: 0.63,0.77 ; \mathrm{p}<0.001$ and $\mathrm{OR}_{\text {run vs jogging }}$ for HS children: 0.93; $95 \% \mathrm{Cl}: 0.87,0.99 ; \mathrm{p}=0.028$ ). Additionally, differences in each group were observed with regard to the footwear independent of the jogging/running condition $\left(\mathrm{OR}_{\text {shod vs barefoot }}\right.$ for $\mathrm{HB}$ children: 1.22; $95 \% \mathrm{Cl}: 1.12,1.33 ; \mathrm{p}<0.001$ and $\mathrm{OR}_{\text {shod vs barefoot }}$ for HS children: $1.08 ; 95 \% \mathrm{Cl}: 1.00,1.16$; $\mathrm{p}=0.043$ ).

A significant age effect was observed for $\mathrm{HB}$ children for the barefoot jogging condition $(\mathrm{OR}=0.83,95 \% \mathrm{Cl}, 0.71$ to 0.96 , $\mathrm{p}=0.011)$ and both running conditions $\left(O R_{\text {barefoot running }}=0.58,95 \%\right.$ $\mathrm{Cl}, 0.50$ to $0.67, \mathrm{p}<0.001 ; \mathrm{OR}_{\text {shod running }}=0.71,95 \% \mathrm{Cl}, 0.62$ to 0.81 ,

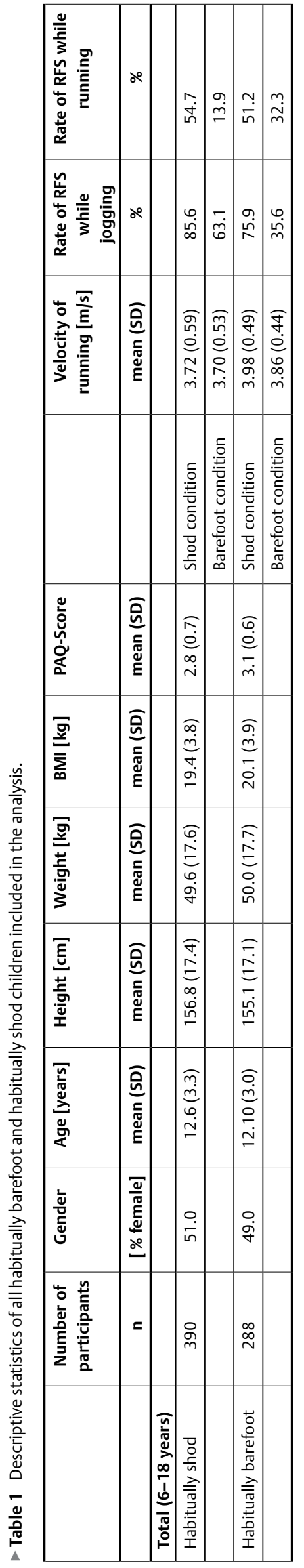




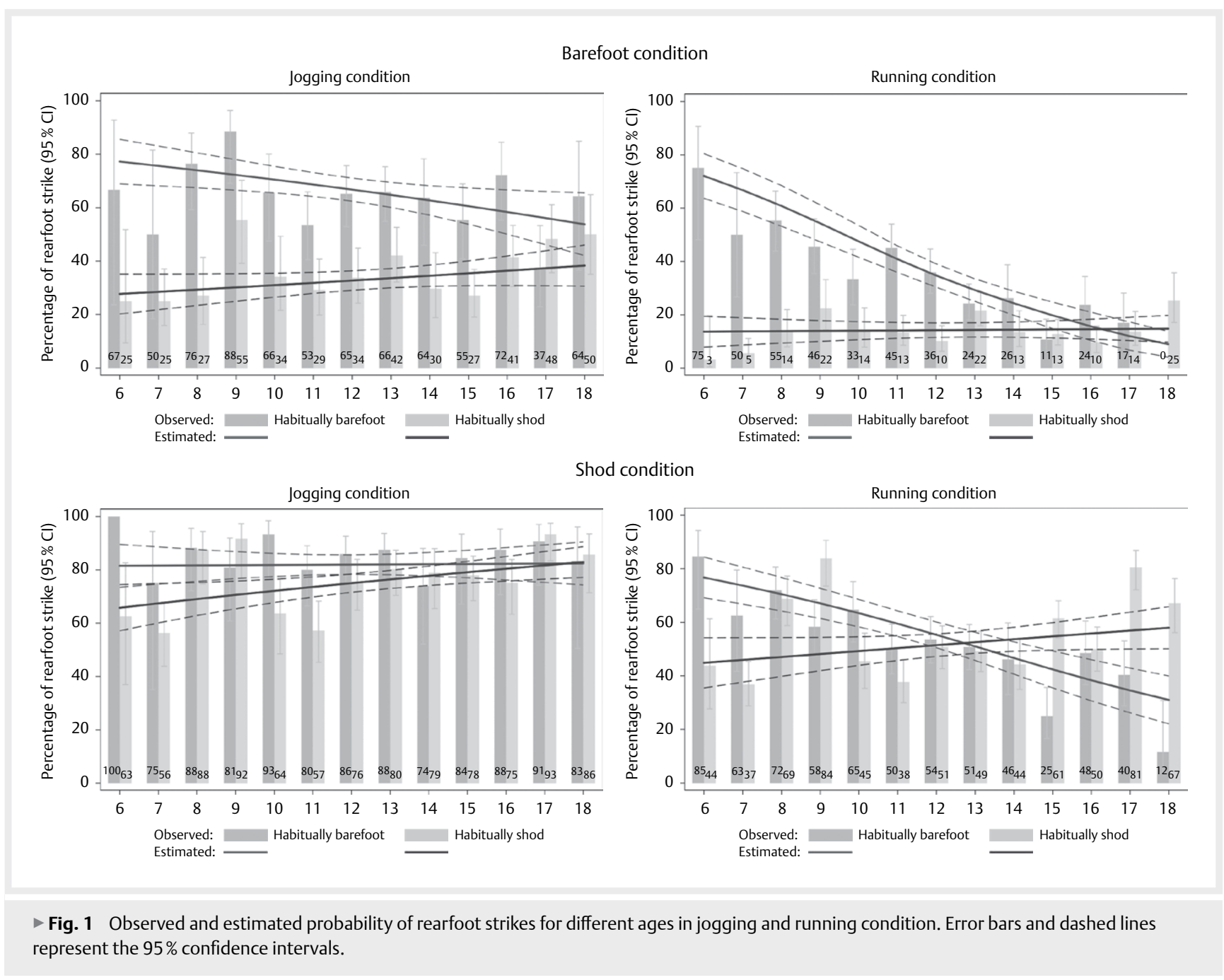

$\mathrm{p}<0.001)$. For HS children, a significant age effect was found only for the shod jogging condition $(\mathrm{OR}=1.18,95 \% \mathrm{Cl}, 1.05$ to 1.32 , $\mathrm{p}=0.006)$. In other words, younger habitually barefoot children were more likely to adopt a RFS pattern when jogging barefoot or running in both conditions, whereas younger HS children were less likely to use a RFS when jogging shod compared to older children. All age effects are shown in > Fig. 2.

\section{Secondary analysis: confounders}

The confounder estimation shows significant effects only for sex $\left(\mathrm{OR}_{\text {female vs male }}=2.28,95 \% \mathrm{Cl} 1.43\right.$ to 3.64, $\left.\mathrm{p}=0.001\right)$, whereas BMI $(\mathrm{OR}=1.03,95 \% \mathrm{Cl}, 0.96$ to $1.11, \mathrm{p}=0.389)$, ethnicity $(\mathrm{OR}=0.42$, $95 \% \mathrm{Cl}, 0.15$ to $1.18, \mathrm{p}=0.102)$ and $\mathrm{PAQ}$ score $(\mathrm{OR}=0.74,95 \% \mathrm{Cl}$, 0.51 to $1.06, p=0.104$ ) did not have a significant effect on the dependent variable FSP.

\section{Discussion}

This was the first study to compare the effects of habituation to barefoot locomotion on FSP in a large cohort of children and adolescents, adding to the limited existing knowledge about the longterm consequences of barefoot locomotion. Overall, children that grow up barefoot showed a higher probability of using a RFS while running barefoot than children who grow up regularly using footwear ( = HS). For HB individuals, the rate of RFS decreases between age 6 and 18 years for most test conditions (barefoot jogging and running, shod running), whereas it remains stable for HS children, except for shod jogging.

\section{Barefoot habituation leads to a variation of foot strike patterns in different populations}

The higher rates of RFS in our HB cohort appear surprising because evidence exists for a reduced rate of RFS and reduced ankle dorsiflexion angles at foot strike in HB runners [16]. To our knowledge, this is the first study to compare FSP between HB and HS children and therefore these findings are difficult to compare. There are a couple of studies investigating FSP of HB adults, comparing them to HS counterparts $[24,25,27]$ and a few reports on adolescents [24, 25]. Lieberman et al. [25] reported a rate of RFS of $12 \%$ in $\mathrm{HB}$ and $62 \%$ in HS Kenyan adolescents running barefoot at a selfselected speed. When comparing the same age levels (11-16 years) to our cohort, we found for the same condition (barefoot running) a rate of RFS of 59-70\% for HB and only $27-43 \%$ for HS adolescents. There are several explanations for the different findings. The Kalen- 


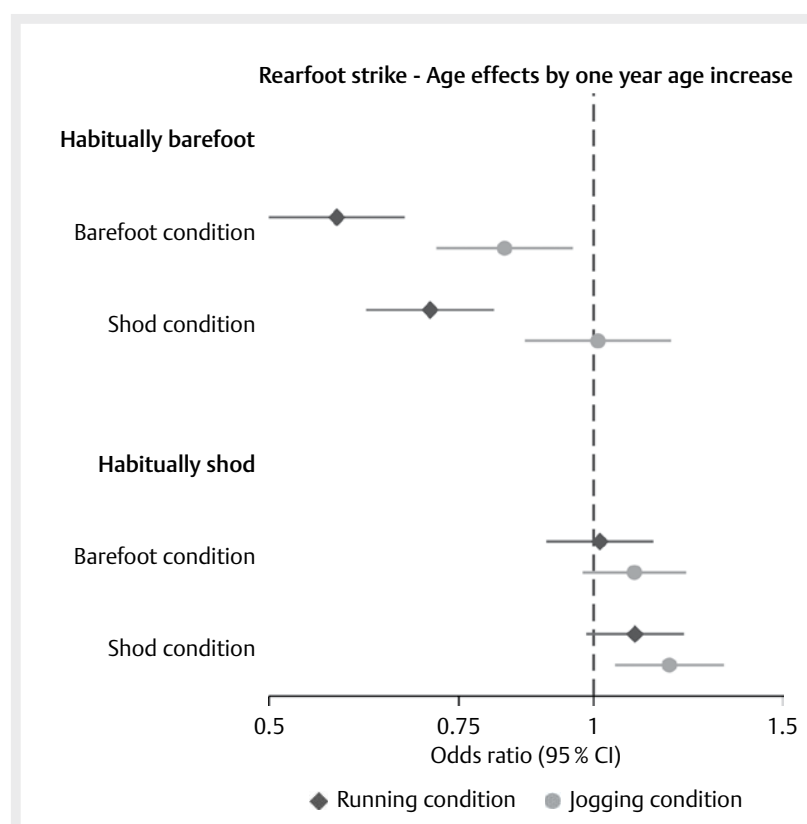

- Fig. 2 Age effects by one year of age increase on the probability of using a rearfoot strike depicted as odds ratio and $95 \%$ confidence intervals. An odds ratio of 1 represents no significant effects. jin runners examined [25] have a large history of successful middle- and long-distance running [20] and therefore, it can be assumed, have a high habituation to competitive running. The South African children in our study are more habituated to other sports that include but do not exclusively consist of running. Furthermore, it is known that the running surface influences foot strike pattern [8], and whereas Lieberman et al. [25] tested on a soft dirt track, our children were primarily tested on asphalt or a hard gymnastic hall floor. However, running on harder surfaces (like in our study) would suggest a higher rate of non-RFS [8], which is not the case. Investigations on other $\mathrm{HB}$ tribes show that RFS might be preferred $[13,33]$. For example, Pontzer et al. [33] report that $86 \%$ of HB Hadza children (age range 5-14 years), not habituated to competitive running, preferably used a RFS when running barefoot at comfortable speed. This is in accordance with our results of $56-88 \%$ probability of rearfoot striking for barefoot jogging in the same age range.

Another factor influencing the FSP seems to be sex. Our confounder effect estimation revealed a higher probability for females to use a RFS. This is in accordance to other findings on HB populations $[24,33]$.

Taking these findings and our results together, the assumption that $\mathrm{HB}$ individuals preferably use a non-RFS to contact the ground might not be correct, especially for juvenile cohorts.

\section{Age effects}

A secondary aim of this study was to evaluate the development of FSP over time. Interestingly, children growing-up barefoot show a higher change in FSP over time, whereas the FSP of HS children remains relatively stable. Since this is the first study to document de- velopment during the early stages of life, it is difficult to compare the data to other studies. Body composition and anthropometry changes constantly during childhood and adolescence $[4,15,30]$, which may influence the ability of the locomotor system to absorb vertical forces during rearfoot running without cushioning. It has been previously shown that running shock attenuation in children is different than in adults [28]. Based on our data, one can speculate that a) younger children may be more predisposed to compensate vertical impact forces during heel landing, and b) (due to their experience) barefoot children are more accustomed to using RFS during barefoot running compared to shod children. Further interaction between foot anthropometrics or motor development may also be possible but will need to be explored in future studies.

\section{Acute effect of changing footwear conditions}

In contrast to limited evidence on long-term effects of barefoot locomotion, there is a large body of literature on the acute effects of barefoot vs. shod running $[6,9,37]$. It is known that changing from shod to barefoot running is typically associated with a higher rate of forefoot strikes and a corresponding reduced rate of ankle dorsiflexion in adults $[9,10,14,25,36]$, adolescents $[24,25]$ and in children [17]. These findings are in accordance with our results for changing footwear conditions for jogging and running and add to the large body of evidence of acute effects of footwear on running biomechanics.

\section{Clinical implications}

Our findings add new elements to the ongoing debate of the advantages and disadvantages of regular barefoot or shod locomotion. It has been widely and controversially discussed whether a certain foot strike pattern in the circumstances of cushioning or non-cushioning is preferable for running performance or injury prevention, with no conclusive evidence $[2,7,22,31]$. Our data show that a transition to forefoot landing cannot be explained only by running without footwear, but is also a result of growing and development during childhood years. Growing up barefoot may help the children to use a RFS during barefoot running more confidently and possibly without the negative influences associated with shock attenuation. This point needs further attention and there is still a need for large prospective studies to shed light on these questions.

\section{Strengths and limitations}

One major strength of this study is that it is the first large cohort study to investigate the effect of habituation to barefoot locomotion on FSP, helping to close the gap in the literature concerning the long-term effects of barefoot locomotion. Although this study had a cross-sectional design, no cause-effect relationship can yet be drawn, which again leads to the demand for well-designed prospective studies. Another limitation might be the non-standardization of footwear during field-testing. The use of the children's own footwear reflects the real-life situation, but it has to be kept in mind that footwear might be a confounding factor in the assessment of foot strike pattern $[9,17,25]$. In contrast to many other studies investigating $\mathrm{HB}$ individuals, this study used a questionnaire to determine whether an individual can be defined as HB. This is a novel approach, but other studies have used different definitions [16]. Therefore, the definition of "habitual barefootedness" needs 
further attention to improve comparability of studies and maybe even to determine a dose-response relationship between the amount of barefootedness and its effects.

\section{Summary}

Foot strike patterns differ between children growing up barefoot and those regularly using shoes. The frequency of RFS in shod and barefoot running is higher in children growing up barefoot, whereas it converges in late adolescence. Together with secondary findings and current literature, it can be concluded that the utilization of FSP is multifactorial and seems to be influenced by footwear habituation, age, sex, speed, ground surface, applied footwear and running experience.

\section{Acknowledgements}

We would like to acknowledge Nicholas R. Dunham for the critical revision of the manuscript. Furthermore we would like to thank all school principals, physical education teachers and finally all pupils that participated voluntarily in this study. This project (Barefoot LIFE) is funded by the Ministry for Science and Research in Hamburg (grant number LFF-FV13).There are no conflicts of interest among any of the authors of this article.

\section{References}

[1] Altman AR, Davis IS. A kinematic method for footstrike pattern detection in barefoot and shod runners. Gait Posture 2012; 35 : 298-300

[2] Altman AR, Davis IS. Prospective comparison of running injuries between shod and barefoot runners. Br J Sports Med 2016; 50 : 476-480

[3] Bonacci ], Saunders PU, Hicks A, Rantalainen T, Vicenzino BGT, Spratford W. Running in a minimalist and lightweight shoe is not the same as running barefoot: a biomechanical study. $\mathrm{Br}$ ] Sports Med 2013; 47: 387-392

[4] de Onis M, Onyango AW, Borghi E, Siyam A, Nishida C, Siekmann J. Development of a WHO growth reference for school-aged children and adolescents. Bull World Health Organ 2007; 85: 660-667

[5] Esculier JF, Silvini T, Bouyer L], Roy JS. Video-based assessment of foot strike pattern and step rate is valid and reliable in runners with patellofemoral pain. Phys Ther Sport 2016, doi:10.1016/j. ptsp.2016.11.003

[6] Franklin S, Grey MJ, Heneghan N, Bowen L, Li FX. Barefoot vs common footwear: A systematic review of the kinematic, kinetic and muscle activity differences during walking. Gait Posture 2015; 42: 230-239

[7] Goss DL, Gross MT. Relationships among self-reported shoe type, footstrike pattern, and injury incidence. US Army Med Dep J 2012; 25-30

[8] Gruber AH, Silvernail JF, Brueggemann P, Rohr E, Hamill J. Footfall patterns during barefoot running on harder and softer surfaces. Footwear Sci 2013; 5: 39-44

[9] Hall JP, Barton C, Jones PR, Morrissey D. The biomechanical differences between barefoot and shod distance running: A systematic review and preliminary meta-analysis. Sports Med 2013; 43: 1335-1353

[10] Hamill ], Russell EM, Gruber AH, Miller R. Impact characteristics in shod and barefoot running. Footwear Sci 2011; 3: 33-40

[11] Harriss DJ, Atkinson G. Ethical standards in sport and exercise science research: 2016 Update. Int J Sports Med 2015; 36: 1121-1124
[12] Hasegawa H, Yamauchi T, Kraemer W]. Foot strike patterns of runners at the $15-\mathrm{km}$ point during an elite-level half marathon. J Strength Cond Res 2007; 21: 888-893

[13] Hatala KG, Dingwall HL, Wunderlich RE, Richmond BG. Variation in foot strike patterns during running among habitually barefoot populations. PLoS One 2013; 8: e52548

[14] Hollander K, Argubi-Wollesen A, Reer R, Zech A. Comparison of minimalist footwear strategies for simulating barefoot running: A randomized crossover study. PLoS One 2015; 10: e0125880

[15] Hollander K, de Villiers JE, Sehner S, Wegscheider K, Braumann KM, Venter R, Zech A. Growing-up (habitually) barefoot influences the development of foot and arch morphology in children and adolescents. Sci Rep 2017; 7: 8079

[16] Hollander K, Heidt C, VDZ BC, Braumann KM, Zech A. Long-term effects of habitual barefoot running and walking: A systematic review. Med Sci Sports Exerc 2017; 49: 752-762

[17] Hollander K, Riebe D, Campe S, Braumann KM, Zech A. Effects of footwear on treadmill running biomechanics in preadolescent children. Gait Posture 2014; 40: 381-385

[18] Hollander K, van der Zwaard BC, de Villiers JE, Braumann KM, Venter R, Zech $A$. The effects of being habitually barefoot on foot mechanics and motor performance in children and adolescents aged $6-18$ years: Study protocol for a multicenter cross-sectional study (Barefoot LIFE project). J Foot Ankle Res 2016; 9: 36

[19] Kowalski K, Crocker P, Donen R. The Physical Activity Questionnaire for Older Children (PAQ-C) and Adolescents (PAQ-A) Manual. Canada: College of Kinesiology, University of Saskatchewan; 2004

[20] Larsen HB, Sheel AW. The Kenyan runners. Scand J Med Sci Sports 2015; 25: (Suppl 4): 110-118

[21] Larson P. Comparison of foot strike patterns of barefoot and minimally shod runners in a recreational road race. J Sport Health Sci 2014; 3: 137-142

[22] Larson P, Higgins E, Kaminski J, Decker T, Preble J, Lyons D, Mclntyre K, Normile A. Foot strike patterns of recreational and sub-elite runners in a long-distance road race. J Sports Sci 2011; 29: 1665-1673

[23] Lieberman DE. What we can learn about running from barefoot running: An evolutionary medical perspective. Exerc Sport Sci Rev 2012; 40: 63-72

[24] Lieberman DE, Castillo ER, Otarola-Castillo E, Sang MK, Sigei TK, Ojiambo R, Okutoyi P, Pitsiladis Y. Variation in foot strike patterns among habitually barefoot and shod runners in Kenya. PLoS One 2015; 10: e0131354

[25] Lieberman DE, Venkadesan M, Werbel WA, Daoud AI, D’Andrea S, Davis IS, Mang'eni RO, Pitsiladis Y. Foot strike patterns and collision forces in habitually barefoot versus shod runners. Nature 2010; 463: 531-535

[26] McCarthy C, Fleming N, Donne B, Blanksby B. 12 weeks of simulated barefoot running changes foot-strike patterns in female runners. Int $]$ Sports Med 2014; 35: 443-450

[27] Mei Q, Fernandez ], Fu W, Feng N, Gu Y. A comparative biomechanical analysis of habitually unshod and shod runners based on a foot morphological difference. Hum Mov Sci 2015; 42: 38-53

[28] Mercer JA, Dufek JS, Mangus BC, Rubley MD, Bhanot K, Aldridge JM. A description of shock attenuation for children running. J Athl Train 2010; 45: 259-264

[29] Mullen S, Toby EB. Adolescent runners: the effect of training shoes on running kinematics. J Pediatr Orthop 2013; 33: 453-457

[30] Muller S, Carlsohn A, Muller ], Baur H, Mayer F. Static and dynamic foot characteristics in children aged 1-13 years: A cross-sectional study. Gait Posture 2012; 35: 389-394

[31] Nigg BM, Baltich J, Hoerzer S, Enders H. Running shoes and running injuries: mythbusting and a proposal for two new paradigms: 'Preferred movement path' and 'comfort filter'. Br J Sports Med 2015; 49: $1290-1294$ 
[32] Perkins KP, Hanney W], Rothschild CE. The risks and benefits of running barefoot or in minimalist shoes: a systematic review. Sports Health 2014; 6: 475-480

[33] Pontzer H, Suchman K, Raichlen DA, Wood BM, Mabulla AZP, Marlowe FW. Foot strike patterns and hind limb joint angles during running in Hadza hunter-gatherers. J Sport Health Sci 2014; 3: 95-101

[34] Santuz A, Ekizos A, Arampatzis A. A pressure plate-based method for the automatic assessment of foot strike patterns during running. Ann Biomed Eng 2016; 44: 1646-1655

[35] Shih Y, Lin KL, Shiang TY. Is the foot striking pattern more important than barefoot or shod conditions in running? Gait Posture 2013; 38 : 490-494

[36] Squadrone R, Gallozzi C. Biomechanical and physiological comparison of barefoot and two shod conditions in experienced barefoot runners. J Sports Med Phys Fitness 2009; 49: 6-13
[37] Tam N, Astephen Wilson JL, Noakes TD, Tucker R. Barefoot running: an evaluation of current hypothesis, future research and clinical applications. Br J Sports Med 2014; 48: 349-355

[38] von Elm E, Altman DG, Egger M, Pocock S], Gotzsche PC, Vandenbroucke JP. Initiative $S$. The Strengthening the Reporting of Observational Studies in Epidemiology (STROBE) statement: Guidelines for reporting observational studies. PLoS Med 2007; 4: e296

[39] Warne JP, Warrington GD. Four-week habituation to simulated barefoot running improves running economy when compared with shod running. Scand J Med Sci Sports 2014; 24: 563-568

[40] Wegener C, Hunt AE, Vanwanseele B, Burns ], Smith RM. Effect of children's shoes on gait: A systematic review and meta-analysis. J Foot Ankle Res 2011; 4: 3 